\title{
The Rat Prefrontostriatal System Analyzed in 3D: Evidence for Multiple Interacting Functional Units
}

\author{
Philippe Mailly, ${ }^{1}$ Verena Aliane, ${ }^{2}$ Henk J. Groenewegen, ${ }^{3}$ Suzanne N. Haber, ${ }^{4}$ and Jean-Michel Deniau ${ }^{5}$ \\ ${ }^{1}$ Institut National de la Santé et de la Recherche Médicale UMRs 952, Centre National de Recherche Scientifique UMR 7224, Université Pierre et Marie \\ Curie, 75252 Paris Cedex 05, France, ${ }^{2}$ Department of Experimental Neurophysiology, Medical Faculty, Ruhr-University of Bochum, D-44780 Bochum, \\ Germany, ${ }^{3}$ Department of Anatomy and Neurosciences, Neuroscience Campus Amsterdam, VU University Medical Center, 1007 MB Amsterdam, The \\ Netherlands, ${ }^{4}$ Department of Neurobiology and Anatomy, University of Rochester School of Medicine, Rochester, New York 14642, and ${ }^{5}$ Institut National \\ de la Santé et de la Recherche Médicale U. 667, Collège de France, 75231 Paris Cedex 05, France
}

Previous studies in monkeys disclosed a specific arrangement of corticostriatal projections. Prefrontal and premotor areas form dense projection fields surrounded by diffuse terminal areas extending outside the densely innervated region and overlapping with projections from other areas. In this study, the mode of prefrontostriatal innervation was analyzed in rats using a 3D approach. Following injections of tracers in defined cortical areas, 3D maps from individual cases were elaborated and combined into a global 3D map allowing us to define putative overlaps between projection territories. In addition to providing a detailed 3D mapping of the topographic representation of prefrontal cortical areas in the rat striatum, the results stress important similarities between the rodent and primate prefrontostriatal projections. They share the dual pattern of focal and diffuse corticostriatal projections. Moreover, besides segregated projections consistent with parallel processing, the interweaving of projection territories establishes specific patterns of overlaps spatially organized along the dorsoventral, mediolateral, and anteroposterior striatal axis. In particular, the extensive striatal projection fields from the prelimbic and anterior cingulate areas, which partly overlap the terminal fields from medial, orbital, and lateral prefrontal cortical areas, provide putative domains of convergence for integration between reward, cognitive, and motor processes.

\section{Introduction}

The striatum is a main component of the basal ganglia involved in adaptive control of behavior and automation of action (Graybiel, 1998, 2005; Yin and Knowlton, 2006). Besides sensory-motor processes underlying motor habits, the striatum contributes to cognitive and motivational processes important for incentivebased learning. Receiving inputs from virtually the entire cerebral cortex and the limbic system (McGeorge and Faull, 1989; Parent, 1990; Groenewegen and Berendse, 1994), the striatum is provided with a diversity of signals (sensory, motor, motivational, emotional) allowing the selection of adapted behavioral goals and elaboration of the corresponding sequence of actions to be engaged. In turn, via the substantia nigra and the pallidum, the striatum influences prefrontal cortical areas implicated in decision making and executive functions (Ilinsky et al., 1985; Groenewegen and Berendse, 1994; Joel and Weiner, 1994;

Received Nov. 12, 2012; revised Jan. 28, 2013; accepted Feb. 17, 2013.

Author contributions: P.M. and J.-M.D. designed research; P.M., V.A., H.J.G., and J.-M.D. performed research; P.M. contributed unpublished reagents/analytic tools; P.M., V.A., H.J.G., S.N.H., and J.-M.D. analyzed data; P.M., H.J.G., S.N.H., and J.-M.D. wrote the paper.

This work has been supported by grants from INSERM, Centre National de la Recherche Scientifique, Universite Pierre et Marie Curie, and Collège de France. Part of this study was financed by a travel grant from Boehringer Ingelheim. We thank Anne-Marie Godeheu and Nicole Quenech'du for technical assistance.

The authors declare no competing financial interests.

Correspondence should be addressed to Philippe Mailly, Institut National de la Santé et de la Recherche Médicale UMRs 952, Centre National de Recherche Scientifique UMR 7224, Université Pierre et Marie Curie, 7-9 quai Saint Bernard, bat B 723, 75252 Paris Cedex 05, France. E-mail: philippe.mailly@snv.jussieu.fr.

DOI:10.1523/JNEUROSCI.5248-12.2013

Copyright $\odot 2013$ the authors $\quad 0270-6474 / 13 / 335718-10 \$ 15.00 / 0$
Middleton and Strick, 1994; Deniau and Thierry, 1997; Haber and Calzavara, 2009).

How the striatum integrates this diversity of information is not fully understood. Rather than an integrative system, most corticobasal ganglia models have emphasized parallel and segregated circuits (Alexander and Crutcher, 1990; Groenewegen and Berendse, 1994; Deniau and Thierry, 1997). However, corticostriatal projections are also characterized by defined patterns of convergence organized both within and between different functional areas. (Yeterian and Van Hoesen, 1978; Alexander and Crutcher, 1990; Reep et al., 2003; Draganski et al., 2006; Haber, 2011). Moreover, in primates, there are two patterns of projections from each cortical area: (1). the well described dense projection fields and (2). diffuse and scattered terminal areas that extend widely outside the densely innervated region. Both these patterns of terminals overlap with those from other cortical regions. The areas of convergent terminals may be important for integration between different aspects of prefrontal functions (Reep et al., 2003; Haber et al., 2006; Calzavara et al., 2007).

Determining the precise mode of convergence and segregation of corticostriatal inputs in rodents is the first step to elucidate the role of striatum in learning behavioral rules in a rodent model. Moreover, comparing the organizational principles between the rodent and primate corticostriatal circuits may contribute to establishing functional homologies across species.

Here, using a 3D approach, we analyzed the organization of prefrontostriatal inputs in the rat brain to determine (1) whether, as in monkeys, the prefrontal cortical areas present focal and 
Table 1. List of animal cases and injection sites

\begin{tabular}{ll}
\hline Cases & Injection \\
\hline 03070703 & DLO (PHAL) \\
08030701 & VLO (PHAL) \\
08030702 & VLO (PHAL) \\
20080702 & VLO (PHAL) \\
90045 & IL (PHAL) \\
25020802 & ACd (PHAL) \\
25020803 & ACd (PHAL) \\
23110601 & ACd (PHAL) \\
14050704 & ACd (PHAL) \\
90278 & ACV (PHAL) \\
19100601 & MOVO (PHAL) \\
14050703 & PL (PHAL) \\
16090901 & PL (PHAL) \\
16090902 & PL (PHAL) \\
18090901 & PL (PHAL) + VLO (FR) \\
18090902 & PL (PHAL) +VLO (FR) \\
01120903 & PL (PHAL)+VLO (FR) \\
94067 & AID (PHAL) \\
90315 & PrCm (PHAL) \\
03070704 & PrCm (PHAL) \\
\hline
\end{tabular}

diffuse terminal fields within the striatum and (2) the degree of overlap between corticostriatal projection fields. The prefrontal cortex of the rat consists of several areas that have been defined based on anatomical and behavioral criteria (Kolb, 1984; Heidbreder and Groenewegen, 2003; Uylings et al., 2003; Schilman et al., 2008; Van De Werd and Uylings, 2008). Injections of tracers were placed in the main subdivisions of the rat prefrontal cortex, corticostriatal projections were charted, and $3 \mathrm{D}$ reconstructions of the projection fields were elaborated. Following a procedure previously applied to investigate the interrelationships between corticostriatal projections fields in monkeys (Calzavara et al., 2007), the 3D maps from individual injection cases were combined into a global 3D map (Mailly et al., 2010).

\section{Materials and Methods}

The organization of prefrontal corticostriatal projections has been examined in experimental cases in which the anterograde tracers Phaseolus vulgaris-leucoagglutinin (PHA-L) injected into areas of the anteromedial, orbital, and rostrolateral cortex. For this study, we selected the cases in which injections were large enough to cover the cortical layers II-VI. Corticocortical and thalamocortical labeling was used to verify the specificity of the injection sites. For each case, the dense (or focal) terminal field was delineated and individual fibers outside the focal projection field (referred to as diffuse projections) were charted throughout the striatum to create $3 \mathrm{D}$ maps of the fields. These were joined into one $3 \mathrm{D}$ map to determine the extent of possible interactions between different prefrontal cortex regions. Reliability of 3D mapping was assessed by comparing the maps of reconstructed projection fields from injections of the same prefrontal cortical area in different animals as well as examining putative convergence between projections in individual cases that had two tracer injections in different cortical regions.

Surgery and tissue preparation. Twenty adult male Sprague Dawley rats (weight 250-350 g; Charles River) were used for the tracing studies (see Table 1). Surgical procedures were applied in strict accordance with the European Communities Council directive 86/609/EEC, 1986. Animals were anesthetized by an injection of sodium pentobarbital (Nembutal, 40 $\mathrm{mg} / \mathrm{kg}$, i.p.; Sanofi). Additional injections of ketamine $(30 \mathrm{mg} / \mathrm{kg}$, i.m.) were occasionally necessary to adjust the level of anesthesia. In addition, $10 \%$ lidocaine was used as local anesthetic for the skin at the sites of incision. Foot withdrawal reflex was checked to assess the depth of anesthesia. The anesthetized animals were placed in a stereotaxic frame. Body temperature was maintained between 36 and $38^{\circ} \mathrm{C}$ by the use of a homeothermic mat. The brain was exposed through small burr holes in the skull, and the anterograde tracer PHA-L (Vector Laboratories) was injected unilaterally in single areas of the prefrontal cortex. In three cases animals received dual injections of PHA-L and Lucifer yellow (LY) (Vector Laboratories) in two different cortical areas (Table 1). The coordinates were derived from the atlas of Paxinos and Watson (1986).

The tracer PHA-L dissolved at $2.5 \%$ in $0.1 \mathrm{~m}$ sodium phosphate buffer (PB), $\mathrm{pH} 7.4$, delivered iontophoretically through glass micropipettes (CG 150; Clark) with an internal tip diameter of $20-30 \mu \mathrm{m}$, by applying positive rectangular pulses ( $7 \mathrm{~s}$ on/7 s off, $5 \mu \mathrm{A}$ for $10-20 \mathrm{~min}$ per injection site). LY was dissolved in $0.1 \mathrm{M} \mathrm{PB}, \mathrm{pH} 7.4$, to yield a $10 \%$ concentration and injected as described above for PHA-L.

After a $7 \mathrm{~d}$ postoperative survival period, the animals were deeply re-anesthetized with sodium pentobarbital (Nembutal, $160 \mathrm{mg} / \mathrm{kg}$, i.p.) and rapidly perfused transcardially with $0.9 \%$ saline followed by a fixative containing $4 \%$ paraformaldehyde- $0.05 \%$ glutaraldehyde in $\mathrm{PB}(0.1 \mathrm{M}$, $\mathrm{pH}$ 7.4). Following an overnight fixation period, the brains were sectioned coronally at $50 \mu \mathrm{m}$ on a freezing microtome and the sections were processed for PHA-L or LY histochemistry. A reliable plane of section was obtained by cutting the brains dorsoventrally at the level of the cerebellum along a vertical plane tilted $18^{\circ}$ toward the rostral part of the brain and sections were cut parallel to this plane. The sections were rinsed with PB followed by $0.05 \mathrm{M}$ Tris/ $\mathrm{HCl}$ (Merck) supplemented with $0.15 \mathrm{M}$ $\mathrm{NaCl}, \mathrm{pH} 7.6$ (Tris buffered saline; TBS-T), and $0.5 \%$ Triton X-100 (TBS-Tx; Merck). They were subsequently incubated for $48 \mathrm{~h}$ at $4^{\circ} \mathrm{C}$ in goat anti PHA-L (1:1000 dilution; Vector Laboratories) or goat anti-LY (1:3000 dilution; Invitrogen) in TBS-T. After rinsing (all intermediate steps between different incubations include three rinses of the indicated buffer for $10 \mathrm{~min}$ each) with TBS-T, the sections were incubated for $18 \mathrm{~h}$ at room temperature in biotinylated rabbit anti-goat IgG followed by incubation in avidin-biotin-peroxidase complex (Vector Laboratories) in TBS-Tx for $1.5 \mathrm{~h}$ at room temperature. After rinsing with Tris/ $\mathrm{HCl}$, PHA-L was visualized by standard diaminobenzidine procedures. Staining was intensified by incubating the tissue for 5-15 $\mathrm{min}$ in a solution of $0.05 \% 3,3^{\prime}$ diaminobenzidine tetra-hydrochloride, $0.025 \%$ cobalt chloride, $0.02 \%$ nickel ammonium sulfate, and $0.01 \% \mathrm{H}_{2} \mathrm{O}_{2}$ to yield a black section product. In cases in which two tracers was injected into a single animal (PHA-L and LY), adjacent sections were treated for each antibody protocol. Sections were mounted onto gel-coated slides, dehydrated, defatted in xylene, and coverslipped with Permount.

Data analysis. Cortical injections limited to superficial or deep layers or in which the tracer had leaked to adjacent areas were not included in the analysis. Injection sites and labeled terminal fields in the striatum were delineated in each experimental case using a light microscope (Laborlux S; Leitz) driven by Neurolucida software (MicroBrightField). Thin labeled fibers containing boutons were charted. Thick fibers without clear terminal boutons as well as fiber bundles were assumed to be passing fibers and were not included. Fiber distributions for each case were charted throughout the rostrocaudal striatum. In the anteromedial cortex there were six injections aimed at the precentral medial ( $\mathrm{PrCm})$, anterior cingulate (ACd and ACv separately), prelimbic (PL), and infralimbic (IL) areas. Three injections in the orbital cortex targeted the medial and ventral (MOVO), ventrolateral (VLO) and dorsolateral (DLO) areas, and finally one injection targeted the dorsal agranular insular (AID) area (Fig. 1). For the anatomical boundaries of these cortical areas we used the detailed anatomical descriptions of Uylings and Groenewegen (Heidbreder and Groenewegen, 2003; Schilman et al., 2008; Van De Werd and Uylings, 2008).

$3 D$ reconstructions. $3 \mathrm{D}$ reconstructions of the focal and diffuse projection fields were created to (1) determine how each projection field occupies the striatum in all directions and (2) develop a global model of the cortical projections fields to compare multiple projections from different cortical regions. Following the method already described by Haber et al. (2006) we used the ability to visualize the dense projections at low magnification to achieve the $3 \mathrm{D}$ rendering of the focal projections. These areas were first delineated using a 2.5 objective and their boundaries were further accurately checked at higher magnification (10 and $20 \times$ ) with the charting of single fibers in the diffuse projection fields. To outline the boundaries of the diffuse projection fields, the labeling in two successive sections was merged. The line diameter of the charted fibers 
was increased and a minimal surface area was defined to threshold automatically the areas of significant density. The same parameters were applied for all cases.

For each case, stained sections were digitized using a scanner (Epson V 750 Pro; Seiko Epson) with a resolution of $3200 \mathrm{dpi}(8 \mu \mathrm{m} / \mathrm{pixel})$ in 16-bit gray scale. A stack of $2 \mathrm{D}$ coronal sections was created using IMOD software (Boulder laboratory for 3D Electron Microscopy of Cells, University of Colorado, Boulder, CO) (Kremer et al., 1996). As previously described by (Mailly et al., 2010) sections were aligned using manual rigid body transformations. The Neurolucida chartings were converted into the IMOD format using a specific program developed for this purpose. A 3D reconstruction that contained structures of interest (striatum, projections fields, cortex, injection sites, and structures of interest such as corpus callosum and anterior commissure) was created for each case separately. Data from each case was then registered into a single reference model using landmarks of key internal structures (striatum and surrounding structures) (Mailly et al., 2010). The model could be sectioned throughout its rostrocaudal extent to determine the patterns of segregation and convergence between projection fields.

\section{Results}

\section{General pattern of projection}

In all cases the terminal field presented the same pattern consisting of focal and diffuse areas. The focal terminal fields that are easily visible at low magnification presented a dense network of fibers and terminals (Fig. 2). The fiber density in the focal projection field is such that charting of individual fibers was precluded. The diffuse projection field expanded the borders of the focal terminal field with a spatial orientation and extension varying with the injected cortical areas. Whereas in some cases the diffuse projection field surrounded in a rather isotropic manner the focal projections, in other cases the orientation was mostly asymmetric and could extend throughout other regions of the striatum. As shown in Figure 3 there was no correlation between the volumes of injection sites and the volumes of focal or diffuse terminal fields. This indicates specificity in the spatial organization of the cortical projections from specific cortical areas. As will be detailed below, this spatial property rules the mode of interaction between cortical projection fields in the striatum.

\section{Distribution of focal projection fields}

Conforming to previous descriptions of prefrontostriatal projections, the focal projection fields were organized as a series of longitudinal bands topographically ordered along the dorsoventral, mediolateral and rostrocaudal axis. The ordering of these bands established a gradient of projections allowing specific patterns of overlaps and segregations (Fig. 4). Starting from the rostromedial part of striatum, the terminal fields from IL (Fig. $4 A$ ) and $\mathrm{ACv}$ (Fig. 4B) terminated in a narrow strip along the medial rim of the nucleus accumbens and the medial dorsal striatum, respectively. Whereas the projections from IL were limited to the shell of nucleus accumbens and the adjacent part of the core (Fig. 4A, columns $\mathrm{z} 1, \mathrm{z} 2$ ), those of ACv were situated dorsally, along the corpus callosum and the lateral ventricle (Fig. 4B). Projections from both IL and ACv ceased to

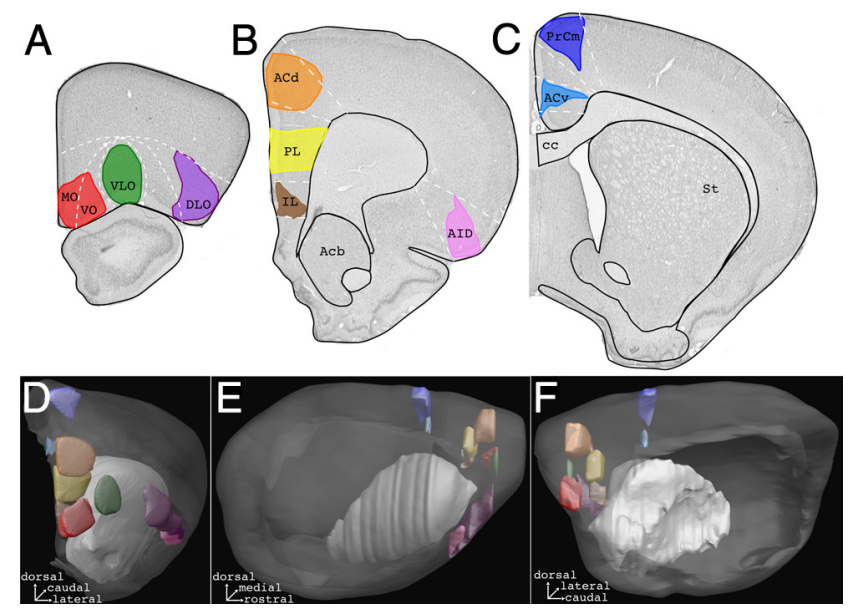

Figure 1. Summary diagram of the anterograde tracer injection sites in the nine cases selected for $3 D$ reconstruction of prefrontostriatal projections. $\boldsymbol{A}-\boldsymbol{C}$, Photomicrographs of coronal Nissl sections with delineation of the maximal extent of tracer. $\boldsymbol{D}-\boldsymbol{F}$, Position and extent of injection sites in the 3D model examined from a rostral view $(\boldsymbol{D})$, lateral view $(\boldsymbol{E})$, and medial view $(\boldsymbol{F})$. Light gray, striatum; dark gray, cerebral cortex. ac, Anterior commissure; Acb, accumbens nucleus; cc, corpus callosum; St, striatum. Color codes: DLO (purple), dorsolateral orbital cortex; VLO (green), ventrolateral orbital cortex; PL (yellow), prelimbic cortex; MOVO (red), medial orbital and ventral orbital cortices; ACd (orange), dorsal anterior cingulate cortex; IL (brown), infralimbic cortex; ACv (cyan), ventral anterior cingulate cortex; AID (magenta), dorsal agranular insular cortex; $\operatorname{PrCm}$ (blue), medial precentral cortex.

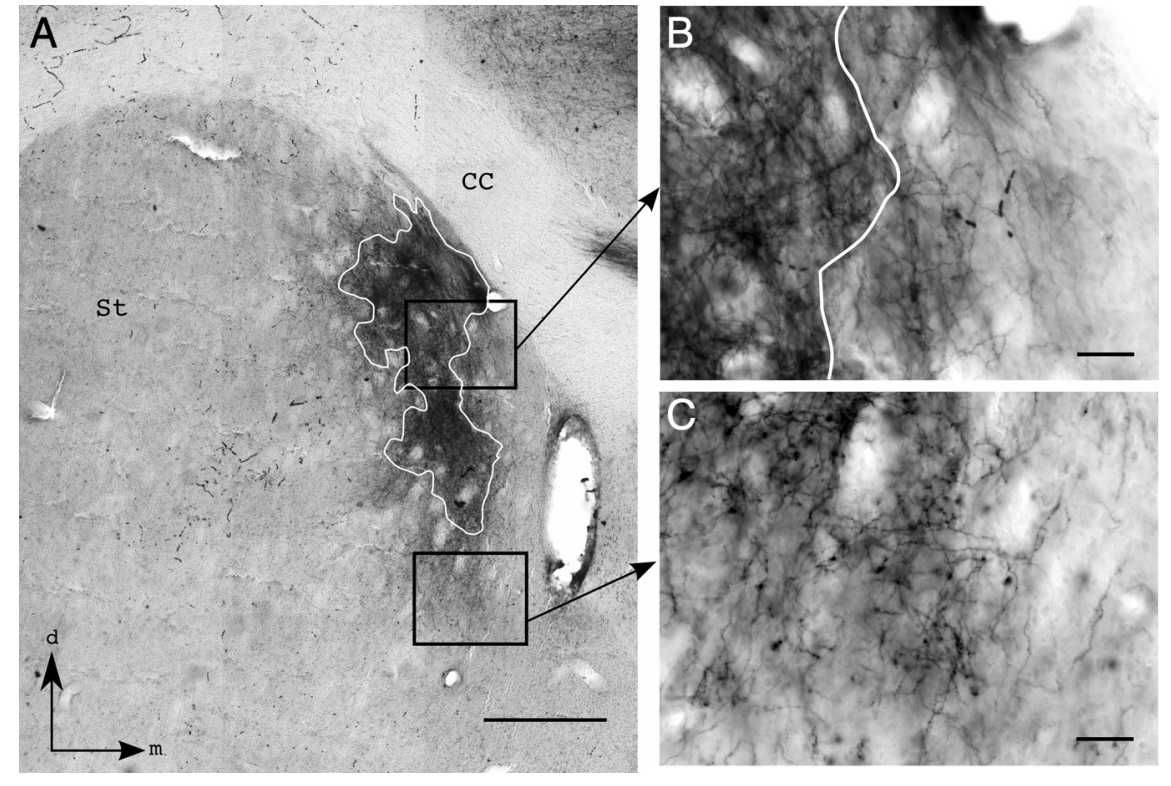

Figure 2. Labeled fibers in the rostral striatum following injection of PHA-L into the prelimbic area. $\boldsymbol{A}, \boldsymbol{B}$, Photomicrographs of the focal and diffuse projections at low magnification and high magnification with the border of the focal projection indicated by a white line. $C$, Photomicrograph at high magnification of the labeled fibers in the diffuse projection area. The two squared areas in $\boldsymbol{A}$ indicate the precise localization of the views illustrated in $\boldsymbol{B}$ and $\boldsymbol{C}$. Scale bars: $\boldsymbol{A}, 500 \mu \mathrm{m} ; \boldsymbol{B}, \boldsymbol{C}, 50 \mu \mathrm{m}$.

exist just rostral to the level of decussation of the anterior commissure (Fig. 4A, B, column z3).

Partly overlapping the IL and ACv projections, the focal terminal field of PL formed a dorsoventrally oriented band occupying quite a large portion of the nucleus accumbens core and shell and the medial part of the dorsal striatum (Fig. 4C). Rostrocaudally, this projection field was among the most extensive of the prefrontostriatal projections studied here, spanning from the rostral pole beyond the level to the decussation of the anterior commissure caudally. Despite an extensive overlap between the 


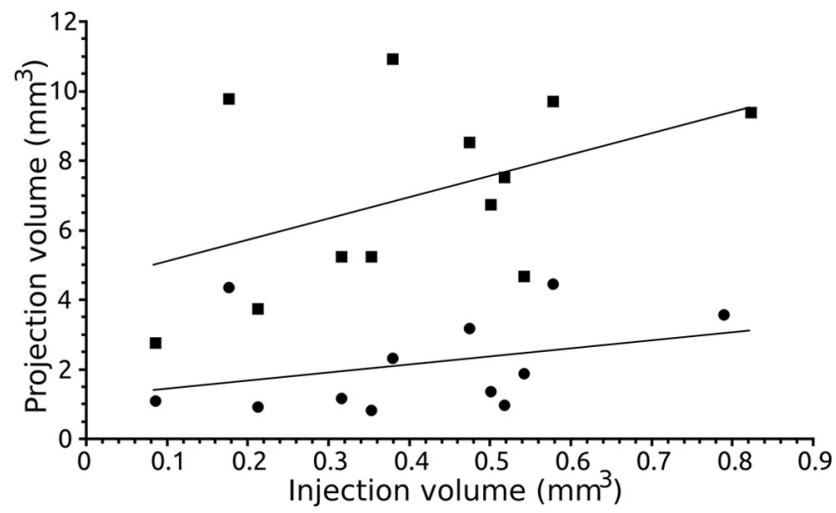

Figure 3. Correlation between the volumes of cortical injection sites and the volumes of striatal projections. Black circles: focal projections $\left(R^{2}=0.117\right.$, Spearman's correlation coefficient $=0.342)$. Black squares: diffuse projections $\left(R^{2}=0.212\right.$; Spearman's correlation coefficient $=0.460$ ).

focal PL projections with those from IL and ACv, they did not share completely the same territory. Compared with IL and ACv, the PL projections extended more laterally and caudally. Moreover, whereas PL projections largely overlapped the IL and $\mathrm{ACv}$ projections at rostral levels (Fig. $4 A-C$, columns z1-3), there was greater separation when progressing caudally. Indeed, in the caudal part of the shell (Fig. 4, column z3), the PL projections avoided the area innervated by IL and, dorsally, PL terminated away from the corpus callosum, avoiding the caudal part of the ACv focal projection field.

Compared with PL, focal projections from VLO, MOVO, and AID occupied a more central position (Fig. $4 A, B, E$ ). VLO and MOVO primarily terminated in the dorsal striatum, although the MOVO projections extended more ventrally into the nucleus accumbens core (Fig. 4B, columns z2, z3). Quite separate from MOVO and VLO territories, AID focal projections were confined to the nucleus accumbens core, encapsulating the anterior commissure (Fig. 4E, column z3). In the dorsal striatum, MOVO and VLO projections converged extensively and partly overlapped the PL focal territory (Fig. $4 A-C$, column z3). However, each cortical projection field extended in separate nonoverlapping zones. In particular, there was a clear mediolateral shift of the focal projection fields progressing from PL to MOVO and, finally, to DLO (Fig. 4A-C, column z3). Here again, segregation was more prominent in the caudal part of the striatum than at rostral levels (Fig. 4 , column z4). Noteworthy, the MOVO and VLO territories were entirely separate from the $\mathrm{ACv}$ and IL focal termination sites. With the exception of some overlap with the PL, AID projections were quite separate from the other components of the prefrontal cortex.

Projections from ACd covered a large territory occupying the centrolateral part of the dorsal striatum and the adjacent dorsolateral region of the core of nucleus accumbens (Fig. 4D). Projections extended from the rostral pole and continued through much of the body of the dorsal striatum, up and including the level of the decussation of the anterior commissure (Fig. $4 D$, column z3). At variance with projections from other prefrontal areas, ACd fibers terminated in a rather discontinuous manner, with large clusters. Medially, these clusters overlapped partly the PL, MOVO, and DLO focal terminal fields but ACd fibers terminated somewhat more laterally.

Finally, DLO and $\mathrm{PrCm}$ focal projections completed laterally and caudally the prefrontal territory (Fig. 4A,E). DLO projections occupied the posterior part of the nucleus accumbens core

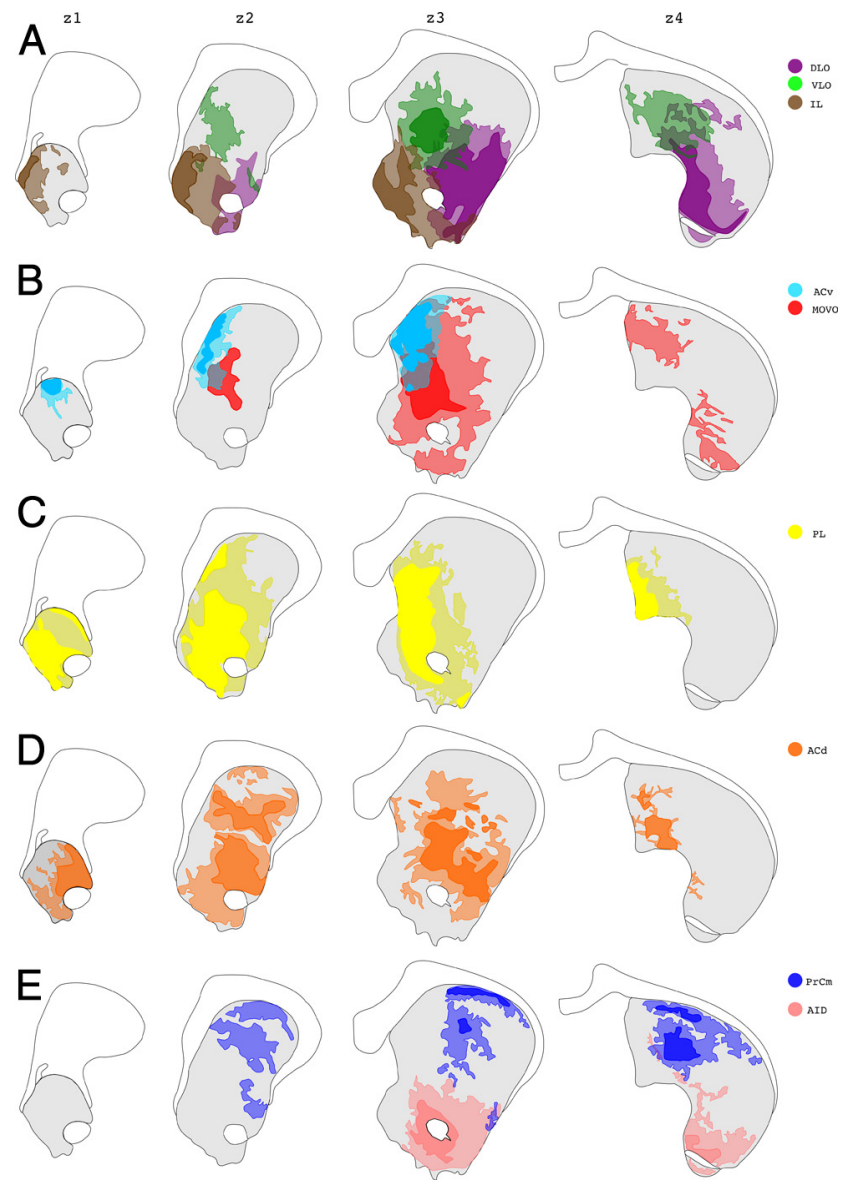

Figure 4. Charts of labeled focal and diffuse terminal fields at different anteroposterior levels of the striatum. Focal terminal fields are presented with dense color and diffuse terminal fields with transparency. $\boldsymbol{A}$, Terminal fields corresponding to DLO (purple), VLO (green), and IL (brown). $\boldsymbol{B}$, Terminal fields corresponding to ACv (cyan) and MOVO (red). $\boldsymbol{C}$, Terminal fields corresponding to PL (yellow). D, Terminal fields corresponding to ACd (orange). $\boldsymbol{E}$, Terminal fields corresponding to $\operatorname{PrCm}$ (blue) and AID (magenta). Abbreviations are as defined in the legend to Figure 1.

and the adjacent dorsal striatum, laterally to the anterior commissure (Fig. 4A, columns z2, z3). Projections extended caudally through much of the body of the striatum, confined along the medial rim of the ventral half of the nucleus. At the opposite, PrCm fibers (Fig. 4E) terminated in the dorsal striatum within two separate focal clusters: one localized centrally and the other dorsolaterally along the corpus callosum. In such a position, PrCm projections interfaced the prefrontal and sensory-motor territories as "barrels" or hindlimb projections located in the dorsal part of the striatum.

The reliability of the reconstruction procedure was assessed by comparing the results of several injection cases performed in the same cortical area. In three cases of successful injections aimed at the PL area, the resulting coincidence of mapping of focal projections in the 3D model have been analyzed using Dice's coefficient (DSC), a superimposition score between different sets of samples (Dice, 1945; Zou et al., 2004). The mean DSC of $0.72 \pm 0.07$ was within the range of good reproducibility (Fig. 5).

\section{Relationships between focal corticostriatal projections}

The 3D reconstructions of the collective focal projections demonstrated potential convergence and segregation between the focal terminal fields arising from the various prefrontal cortical 
areas. As illustrated in Figures 4 and 6, none of the focal projections from the various prefrontal areas occupied a completely separate territory. A significant volume of each dense projection field (30-40\% for DLO, IL, and ACv; 63-96\% for VLO, PL, MOVO, ACd, and AID) was occupied by other cortical areas (Fig. 6D, Table 2). The main exception to this pattern of considerable overlap was formed by the PrCm, which displayed greater segregation (only $5 \%$ overlap with other territories of focal projections). Thus, throughout its extension along the different axis of the striatum, the PL focal projections overlapped with part of the dense projection fields from almost all (except PrCm) other prefrontal areas. Among the territories overlapped by PL, those of MOVO, ACd, IL, ACv, and AID received the most important proportion of focal PL projections (Fig. 6D). A rather similar situation applied for the ACd and MOVO in the dorsal striatum. ACd projections partly overlapped DLO, VLO, PL, and MOVO focal projection fields whereas the MOVO projection overlapped with a substantial part of VLO, PL, ACd, and $\mathrm{ACv}$ projections. In the case of DLO, its projections also occupied part of different prefrontal projections in the dorsal and ventral striatum, the more significant overlaps focused onto ACd and AID. As for IL, ACv, AID, and PrCm, their projection fields occupied only very limited portions of other focal projection fields.

As a consequence of the topography of corticostriatal projections, interfaces between specific cortical projections did not extend throughout the striatum but were rather confined to selective rostrocaudal levels. Moreover within each rostrocaudal level, convergence between cortical projections was located in restricted regions.

\section{Dorsal striatum}

In the rostral part of the dorsal striatum, PL and ACd focal projections occupied quite a large portion of the nucleus, except for its dorsolateral quadrant (Figs. 4C,D, 6, columns z2, z3). PL and ACd terminals converged in a central region, dorsal to the anterior commissure. Compared with ACd, the PL focal area extended more medially and dorsomedially within the ACv territory. At this rostral level of the dorsal striatum there was no focal projection from the orbital and AID areas. Slightly more caudally, the PL and ACd projections showed a tendency to segregate mediolaterally. At this level, PL projections converged with ACv, MOVO, and VLO in a large centromedial region. Compared with PL, MOVO and VLO projections extended more laterally where they overlapped with the ACd territory (Figs. 4, 6). Although VLO and MOVO shared partly the same territory, focal projections from VLO were located more laterally, allowing a specific territory of convergence with ACd projections. In the posterior part of the rostral striatum, just rostral to level of the decussation of anterior commissure, the projections of DLO appeared. They occupied a ventrolateral quadrant mostly segregated from those of PL, MOVO, $\mathrm{VLO}$, and ACV. In contrast, DLO projections converged with those of ACd. At the level of the decussation of anterior commissure there were no more focal projections of $\mathrm{ACd}, \mathrm{ACv}$, and MOVO. There PL, VLO, and DLO occupied primarily separate regions. In contrast there was potential convergence between projections of VLO and PrCm.

\section{Nucleus accumbens}

In the nucleus accumbens, the $3 \mathrm{D}$ reconstructions showed a major segregation between IL, ACd, MOVO, AID, and DLO (Figs. 4, 6 , column $\mathrm{z} 1$ ). In contrast major overlaps were potentially evident between PL and IL medially and between PL and ACd centrally and dorsally to the anterior commissure. In more 

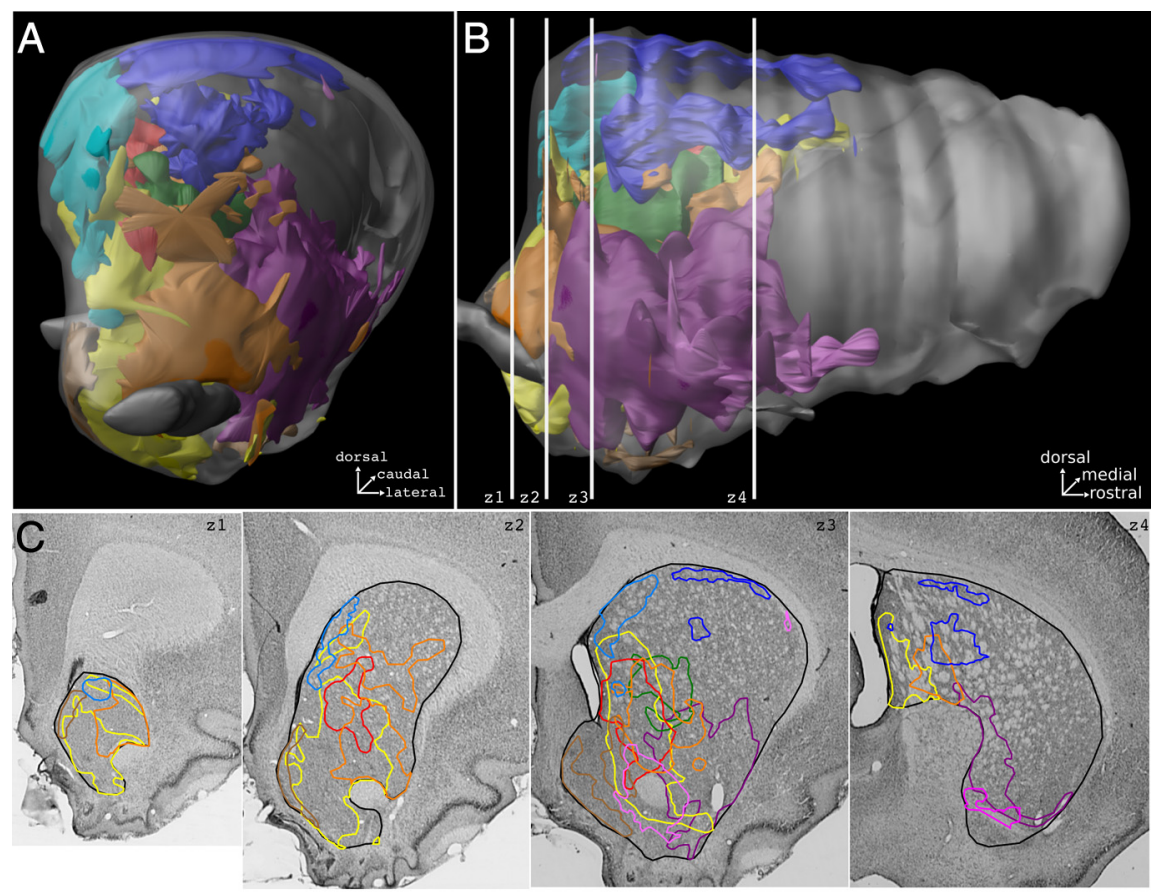

D
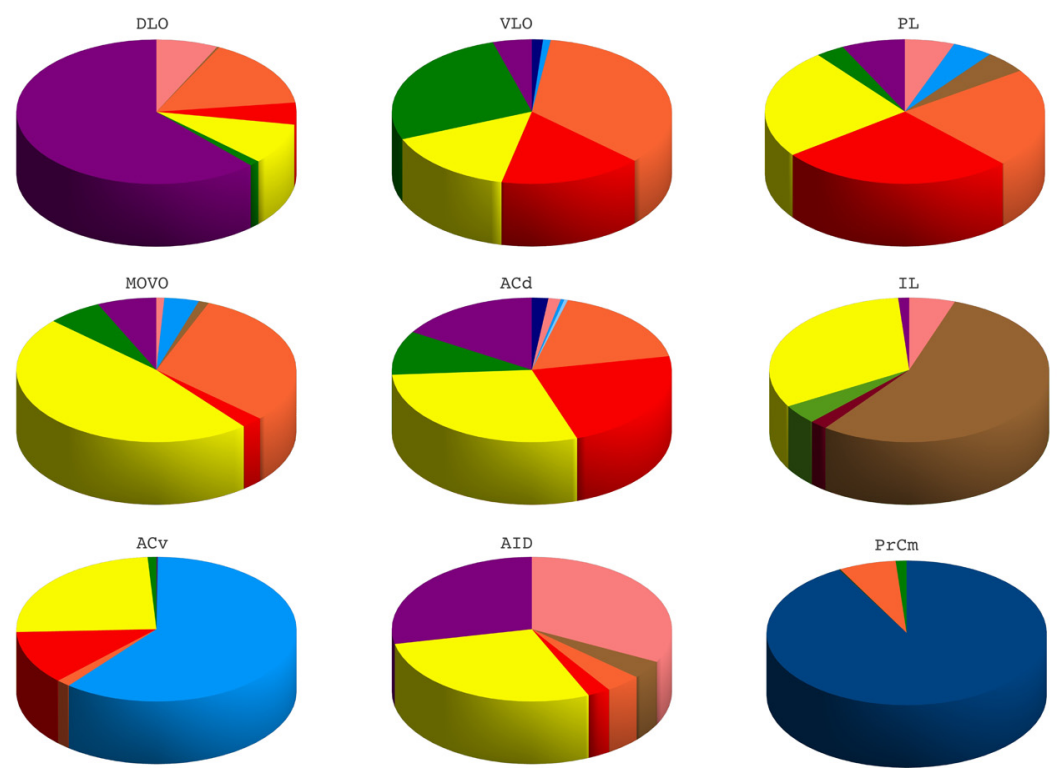

Figure 6. 3D renderings of the focal corticostriatal projection fields from the nine prefrontal areas studied and calculations of their volumes of overlap. $\boldsymbol{A}, \boldsymbol{B}$, Frontal and lateral view of the $3 \mathrm{D}$ global model of striatum showing all focal projections of prefrontal cortex areas studied. White lines in $\boldsymbol{B}$ indicate the level where the coronal sections of the global model illustrated in $\mathbf{C}(\mathrm{z} 1, \mathrm{z2}, \mathrm{z} 3$, and z4) were performed. C, Coronal slices throughout the 3D model with their corresponding Nissl sections ordered from rostral ( $\mathrm{z} 1)$ to caudal (z4). D, Quantification of the volume of overlaps between focal projections. In each case, the portions of the pie chart represent the amount of the focal projection field from a given cortical area that is overlapped by the focal projection fields from the other cortical areas. The portion of the pies represented with the color code of the reference area indicates the part of the focal projection field remaining segregated from the focal projection fields of the other areas. For example, in the case of DLO pie (purple code), the purple pie part represents the portion of DLO projection that is segregated from all the other projections; the magenta pie part represents the portion of DLO overlapped by the AID projection; the orange pie part represents the portion of DLO overlapped by the ACd projection; the red pie part represents the portion of DLO overlapped by MOVO projections; the yellow pie part represents the portion of DLO overlapped by PL; and the green pie part represents the portion of DLO overlapped by VLO. Note the lack of complete overlap. Color codes: DLO (purple), VLO (green), PL (yellow), MOVO (red), ACd (orange), IL (brown), ACv (cyan), AID (magenta), and $\operatorname{PrCm}$ (blue). Abbreviations are as defined in the legend of Figure 1.

caudal parts of the core of nucleus accumbens additional convergence was potentially found between PL and AID around the anterior commissure and between $\mathrm{ACd}$ and DLO ventrolaterally.
Distribution of diffuse projection fields In each injection case, a large number of labeled fibers bearing boutons extended outside of the focal terminal fields (Fig. 2). These fibers were individually charted and 3D maps were reconstructed and superimposed to the focal projections. Besides fibers surrounding focal areas, fibers and clusters of terminals were also observed in very distant striatal regions invading the focal and diffuse projections from other cortical areas. As illustrated in Figures 4 and 7 and Table 2, the extension of the diffuse projection fields differed with the injected cortical areas. In the case of $\mathrm{ACv}$ the diffuse projections remained in the vicinity of the dense projection field producing a modest increase in the volume of projection territory (factor of 1.3). In contrast, in most of the other cases, diffuse projections were more extensive and provided a very significant increase in the volumes of projection fields, ranging from 2 in the case of IL to 7.7 in the case of AID (Table 2).

In terms of spatial arrangement, the distribution of diffuse projections around the dense field was clearly anisotropic, the main directions of extension varying with the injected cortical area (Fig. 4). In the case of VLO and PrCm, the diffuse projections extended mostly rostrocaudally and remained in the central region of the dorsal striatum occupied by the dense projection field. In the case of MOVO, PL, and $\mathrm{ACd}$, the diffuse projections extended considerably dorsoventrally and laterally from the dense field, spreading out from the dorsal part of the dorsal striatum to a large sector of the core of nucleus accumbens. In the case of IL and AID, projections remained in the nucleus accumbens but extended considerably laterally to the dense field. Thanks to its diffuse projections, the IL territory spread out from the shell throughout the core of nucleus accumbens, up to the anterior commissure. Similarly, the AID territory, in which the dense field was confined around the anterior commissure, showed a considerable extension toward the most lateral part of the nucleus accumbens.

Such an expansion of corticostriatal projection fields provided by diffuse innervation strengthened and enriched the patterns of interactions between prefrontostriatal projections. Accordingly, comparison of Figures $6 D$ and $7 D$ illustrate the remarkable increase in the volumes of projection fields of individual cortical areas overlapped by projections from other cortices and the reduction in the volumes of projection fields occupied by completely separate areas. Whereas the volume of the focal projection fields occupied by a completely 
separate cortical area reached a mean value of $47.9 \%$ when considering only the overlaps between focal projections, this value dropped to $9.05 \%$ after incorporating in the model the diffuse innervations (Table 2).

In most cases, the diffuse projections strengthened the rules of corticostriatal interactions existing between the focal projection fields, just by increasing the volume of overlaps. However, due to differences in the extension and spatial organization of diffuse projections, they modified the relative contribution of the areas involved in the corticostriatal overlaps. Taking the VLO focal territory as an example, whereas the ACd focal field was the main extrinsic source overlapping the VLO focal field, when including the diffuse projection, the relative contribution of ACd was reduced in favor of the contribution of DLO, MOVO, and PL (Figs. 6D, 7D). In addition to strengthening the patterns of focal corticostriatal interactions, diffuse projections provided some additional patterns of potential convergence. In the dorsal striatum, such additional interactions involved overlaps between the diffuse projections from ACd, VLO, MOVO, PL, and DLO onto the focal projection field of PrCm. Also, the focal ACv projection was partly overlapped by diffuse projections from VLO and ACd. Finally, in the nucleus accumbens, there was overlap between the diffuse projection fields from MOVO, ACd, and AID onto the dense projections field from IL and reciprocally, the diffuse projections from IL overlapping part of the dense projection fields from the other areas.

Individual cases in which two tracer injections were performed in separate cortical areas, illustrate the convergence between diffuse and focal projection fields. In three cases, injections of PHA-L were placed in PL and injections of LY in VLO. As expected from the 3D model, diffuse LY labeling from VLO was observed in the focal projection field labeled with PHA-L (Fig. 8).

As mentioned above, due to the spatial arrangement of dense and diffuse projection fields, interfaces between corticostriatal projections presented specificity in terms of cortical areas and striatal regions. When combining the diffuse and focal projections from each prefrontal area within a single 3D model, it appears that the multiple combination of overlaps subdivide the prefrontostriatal territory into a complex 3D mosaic of subterritories defined by their specific combination of inputs.

\section{Discussion}

\section{The dual corticostriatal} projection pattern

Extending previous observations in primates (Calzavara et al., 2007) and rodents (Wright et al., 1999), corticostriatal pro-

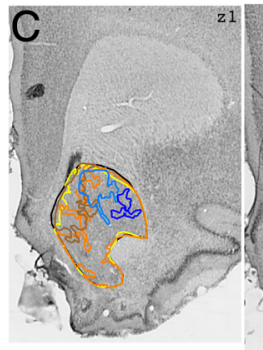

D
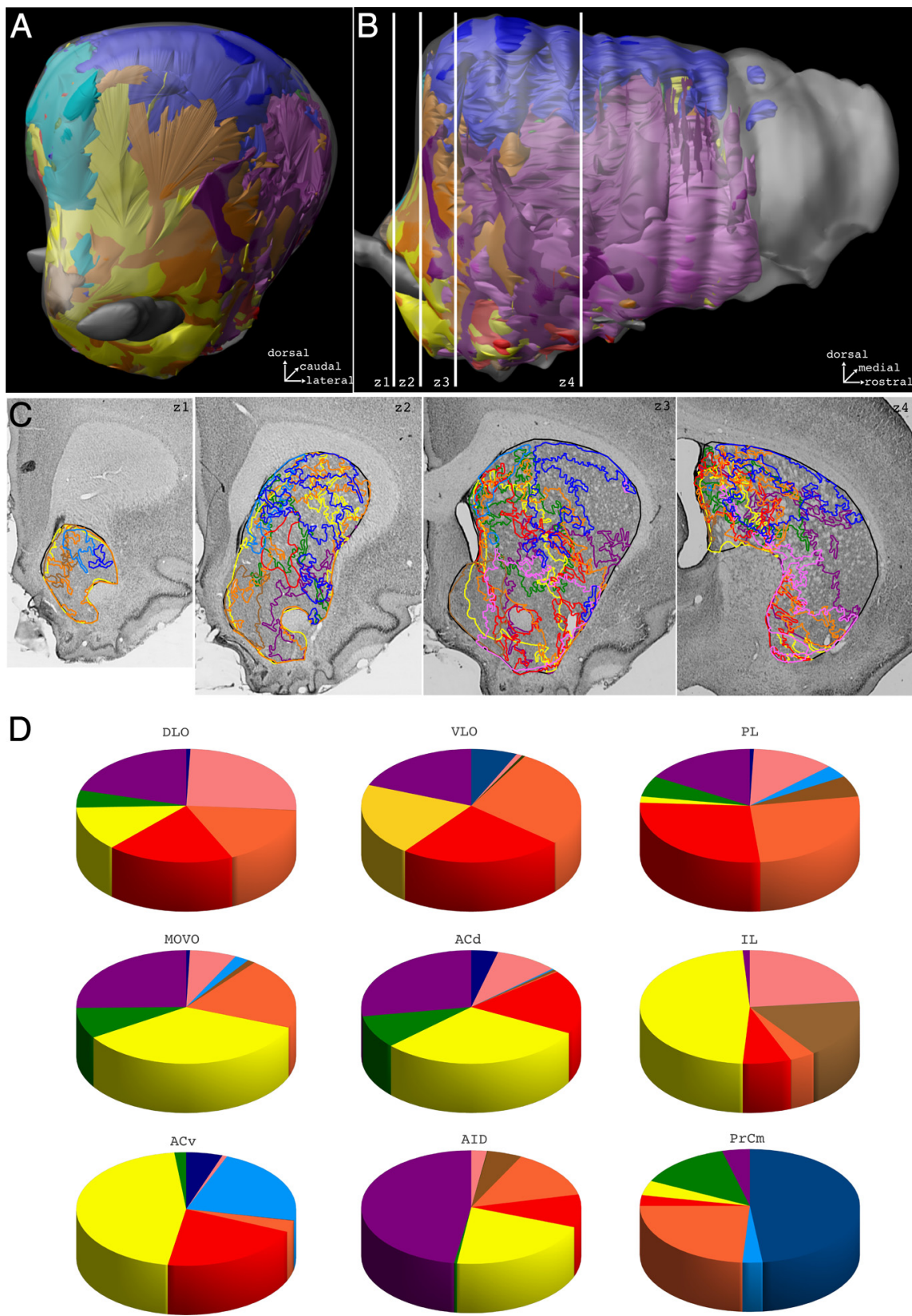

Figure 7. 3D renderings of diffuse projection fields and calculations of their volumes of overlap. $A, B$, Frontal and lateral view of the striatum showing all diffuse projections from prefrontal cortex areas studied. White lines in $\boldsymbol{B}$ indicate the level of coronal sections of the global model illustrated in $\mathbf{C}(z 1, z 2, z 3$, and z4). C, Coronal slices throughout the 3D model with their corresponding Nissl sections. D, Quantification of the volumes of the focal projection from a given cortical area overlapped by the entire projection field (including focal and diffuse) from the other cortical areas. Note the very limited portion of the projection remaining segregated from others. Color codes: DLO (purple), VLO (green), PL (yellow), MOVO (red), ACd (orange), IL (brown), ACv (cyan), AID (magenta), and $\operatorname{PrCm}$ (blue). Abbreviations are as defined in the legend of Figure 1. 


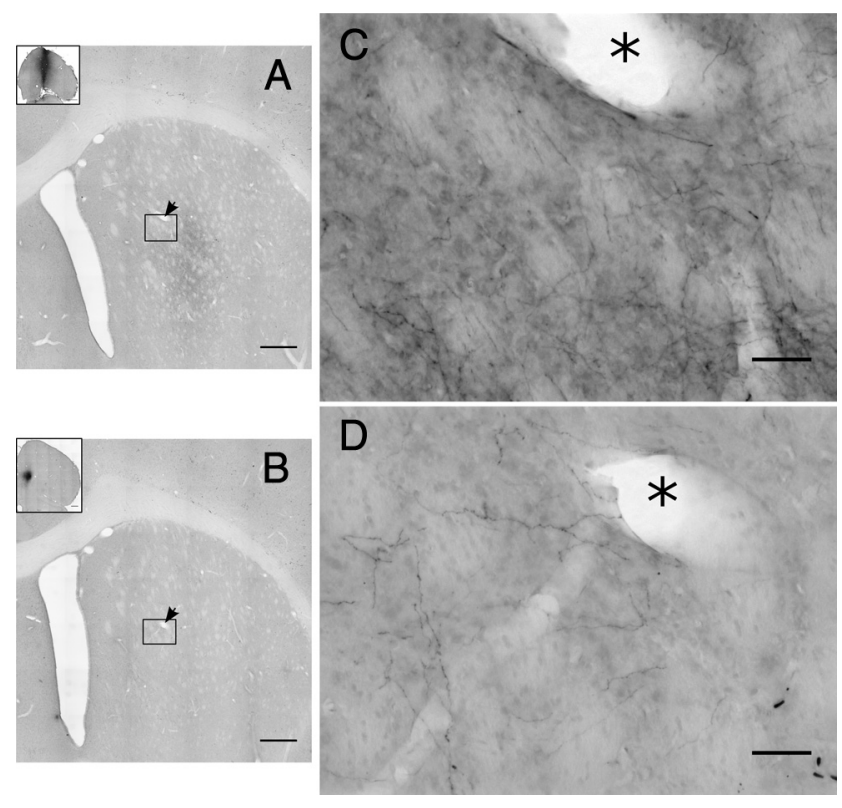

Figure 8. Photomicrographs of two adjacent coronal sections showing labeled fibers in corresponding striatal regions following dual cortical injections of the anterograde tracers $L Y$ in VLO and PHAL in PL areas. (insets $\boldsymbol{A}$ and $\boldsymbol{B}$ ). Tracers were revealed in adjacent sections $(50 \mu \mathrm{m})$. $A, \mathrm{LY}$ injection in VLO area. $\boldsymbol{B}, \mathrm{PHAL}$ injection in PL area at low magnification. $\boldsymbol{C}, \boldsymbol{D}$, High magnification of the square areas in $\boldsymbol{A}$ and $\boldsymbol{B}$. Arrows in $\boldsymbol{A}$ and $\boldsymbol{B}$ point at a common blood vessel indicated with a star in $\boldsymbol{C}$ and $\boldsymbol{D}$. Single PHAL-labeled fibers were found in the central part of striatum that contain focal and diffuse VLO projections (C, D). Scale bar, $A, B, 500 \mu \mathrm{m}$; C, D, $50 \mu \mathrm{m}$.

jection fields from the rat prefrontal areas were found to comprise two components: a focal termination site characterized by a very high density of labeled fibers and a more diffuse zone of labeled fibers surrounding the focal projection. Such pattern of labeling did not simply result from a decreased strength of labeling due to a gradient of tracer concentration at the injection site. Indeed, the relative extension of the dense and diffuse areas were not related to the extent of the injection sites but appeared primarily related to the injected cortical area. Such dual mode of corticostriatal innervation is reminiscent of the patterns of axonal arborizations observed at a single cell level with some corticostriatal neurons providing a high density of terminals in a small striatal volume whereas others extend a low background innervation (Kincaid and Wilson, 1996; Zheng and Wilson, 2002). These two modes of corticostriatal innervation appear to originate from distinct cortical layers. Here to reveal the maximal extent of corticostriatal projections, we selected injection cases covering most of the cortical thickness. As will be discussed below, such a dual organization of corticostriatal projections allows the striatum to operate different modalities of integration of cortical inputs. In addition to maintaining a high level of specificity thanks to a rigorous topography, both patterns of focal and diffuse terminals overlap with those from other cortical regions generating hot spots of integration in which inputs of different strength and different nature coalesce.

\section{The focal pattern of corticostriatal projections}

Medium-sized spiny neurons (MSNs), the main population of striatal output neurons, are characterized by nonlinear membrane properties allowing a filtering of small and uncorrelated synaptic events. This property is due to voltage-gated potassium currents activated in the subthreshold range of potentials (Nisenbaum and Wilson, 1995; Kasanetz et al., 2008). Conse- quently, at rest MSNs are highly hyperpolarized and poorly reactive to depolarizing inputs. They need strong and correlated synaptic inputs to discharge (Calabresi et al., 1987; Nisenbaum and Wilson, 1995). As a powerful source of inputs potentially able to drive the MSNs, the focal corticostriatal projections possess an important functional status.

The topographical arrangement of corticostriatal projections has been the focus of much interest since it allowed approaching the functional organization of striatum, a structure otherwise characterized by a homogeneous cytoarchitecture. In accordance with previous studies in rats, focal projection fields from prefrontal cortical areas distributed along longitudinal striatal zones with a slightly oblique dorsomedial to ventrolateral orientation. This gradient of cortical inputs fits with the proposed functional subdivision of the striatum into limbic, associative, and sensorymotor domains along a ventromedial to dorsolateral axis (Voorn et al., 2004). Evidences suggesting a segregated processing of cortical inputs within the striatum and the subsequent relay stations of striatal output pathways led to the concept of a parallel architecture of the corticobasal ganglia circuits (Alexander and Crutcher, 1990; Berendse et al., 1992a,b). Certainly significant for this concept is the ordered distribution of focal corticostriatal projections. They form a series of longitudinal bands occupying distinct mediolateral, dorsoventral, and rostrocaudal position. This allows segregation of defined prefrontal projections occupying complementary position in the corticostriatal map (Voorn et al., 2004). However, segregation of corticostriatal inputs is only one facet of striatal information processing. As shown here by elaboration of $3 \mathrm{D}$ models and in agreement with observations in monkeys, we provide evidence for an additional level of organization based on specific overlaps between prefrontal corticostriatal projection territories. As illustrated in Figure 6, $C$ and $D$, none of the focal projections from the various prefrontal areas occupied a completely separate territory. In the course of their extension along the dorsoventral, mediolateral, and rostrocaudal axis, the focal projection field of each cortical area partially overlapped the projection fields of other areas. This was particularly impressive in the cases of the PL and ACd, which occupied a large territory within the dorsal and ventral striatum overlapping part of the focal projections of most other prefrontal areas. Due to the topographic arrangement of cortical projections, the modalities of overlaps vary across the different striatal axes and determine a mosaic of subterritories allowing combinations of multiple signals from the different prefrontal cortical areas. Noteworthy, the overlap between focal projection fields from different cortical areas was more prominent in the rostral part of the caudateputamen than in the caudal half. In agreement with earlier reports in monkeys (Calzavara et al., 2007; Roberts et al., 2007), this organization places the head of the caudate nucleus in the position of "hot spot" for the integration of information from functionally different frontal cortical areas.

In previous conceptual models of the striatal functional compartmentalization, the extension of individual corticostriatal projection zones across the dorsal and ventral striatum was emphasized. This led to challenging the classical dorsoventral divide of the striatum (Voorn et al., 2004). Indeed, projections from PL and ACd encompass the dorsal and ventral striatal subdivisions (Berendse et al., 1992; Voorn et al., 2004; present study). However, a number of other cortical areas (IL, MOVO, ACv, PrCm) occupy a more limited dorsoventral territory and distinguish the integrative processes performed in ventral and dorsal parts of the striatum (Schilman et al., 2008; present study). Functionally, the striatum is engaged in appetitive behavior to select actions 
based on their predicted reward value. As documented by behavioral approach, whereas the ventral striatum, in particular the nucleus accumbens, is necessary for the acquisition of pavlovian appetitive responses and contribute to the motivational control of instrumental performance, the dorsal striatum is critical for the acquisition of goal-directed behaviors controlled by actionoutcome contingencies and in the formation of stimulus response underlying the habits. Therefore, in addition to the spiraling mechanism established by the striato-nigral-striatal circuit (Haber et al., 2000), the cortical areas such as PL and ACd, in which projections span the two striatal subdivisions, offer a link between the different learning processes during the acquisition of a new task.

In a phylogenetic perspective, the mode of convergence between prefrontostriatal projections documented here stress common features between the organization of the rodent and primate prefrontostriatal system. First, the orbitofrontal areas, which provide an important source of reward signals to the striatum, occupy a large part of the medial and central region of the dorsal striatum. Each of these areas encodes specific aspects of rewardrelated information such as magnitude and probability of reward. As in monkeys, focal projection fields from distinct orbitofrontal areas presented important areas of overlap indicating that in rats as in primates, the dorsal striatum constitutes a major site for the coordination of these processes. In monkeys, the reward-related part of the striatum defined by the projection territory of the orbitofrontal cortex and ACd shares important convergent areas with the projection field of the lateral prefrontal cortex. Rats have no dorsolateral prefrontal cortex but the PL area, which is critical for attentional processes, behavioral flexibility, and the encoding of action outcome, shares some of the cognitive functions devoted to the monkey dorsolateral prefrontal areas (Uylings et al., 2003). Interestingly, the PL projections occupied a large volume within the dorsal striatum and showed important zones of overlap with the orbitofrontal and ACd projections. These observations generalize to the rodent the concept viewing the dorsal striatum as a major site for the integration of different aspects of reward and cognition making it a central site for the development of incentive-based learning.

\section{The diffuse patterns of corticostriatal projections}

In each case, the dense projection fields were surrounded by diffuse projections, which expanded the influence of cortical areas onto the neighboring corticostriatal territories. Considering the nonlinear membrane properties of MSNs, which require a strong excitatory input to be depolarized from a resting state (Calabresi et al., 1987; Nisenbaum and Wilson, 1995; Kasanetz et al., 2008), it is unlikely that the diffuse projections provide such efficient input on their own. However, when discharged, MSNs become able to integrate small synaptic inputs. In this situation, signals from the diffuse projection fields may contribute to the elaboration of the striatal output signal. Besides MSNs, the striatal network comprises several populations of interneurons that are activated by corticostriatal afferents (Mallet et al., 2005; Fino et al., 2008, 2009). In particular, the population of fast-spiking GABAergic interneurons exerts a strong shunting effect on the synaptic excitatory inputs of MSNs and contributes to contrast effects between neighboring MSNs via a feedforward inhibitory mechanism (Koós and Tepper, 1999; Planert et al., 2010). Altogether, the organization of the corticostriatal projections and the physiological properties of the neuronal elements suggest a dual functional mode of the diffuse projections. Depending on the distribution of cortical activity in time and space, the combina- tion of focal and diffuse projections may either contribute to facilitate the activation of MSNs in some spots of convergence and, conversely, inhibit MSNs in others, exerting contrast effects. Therefore, the combination of focal and diffuse corticostriatal projections may provide a very dynamic network allowing us to precisely select the striatal output neurons to be activated. Such a dynamic network allows multiple and specific interactions between corticostriatal inputs, which amplifies the combinatorial power of the associations between them.

\section{References}

Alexander GE, Crutcher MD (1990) Functional architecture of basal ganglia circuits: neural substrates of parallel processing. Trends Neurosci 13:266271. CrossRef Medline

Berendse HW, Galis-de Graaf Y, Groenewegen HJ (1992a) Topographical organization and relationship with ventral striatal compartments of prefrontal corticostriatal projections in the rat. J Comp Neurol 316:314-347. CrossRef Medline

Berendse HW, Groenewegen HJ, Lohman AH (1992b) Compartmental distribution of ventral striatal neurons projecting to the mesencephalon in the rat. J Neurosci 12:2079-2103. Medline

Calabresi P, Misgeld U, Dodt HU (1987) Intrinsic membrane properties of neostriatal neurons can account for their low level of spontaneous activity. Neuroscience 20:293-303. CrossRef Medline

Calzavara R, Mailly P, Haber SN (2007) Relationship between the corticostriatal terminals from areas 9 and 46, and those from area $8 \mathrm{~A}$, dorsal and rostral premotor cortex and area 24c: an anatomical substrate for cognition to action. Eur J Neurosci 26:2005-2024. CrossRef Medline

Deniau JM, Thierry AM (1997) Anatomical segregation of information processing in the rat substantia nigra pars reticulata. Adv Neurol 74:83-96. Medline

Dice L (1945) Measures of the amount of ecologic association between species. Ecology 26:207-302. CrossRef

Draganski B, Gaser C, Kempermann G, Kuhn HG, Winkler J, Büchel C, May A (2006) Temporal and spatial dynamics of brain structure changes during extensive learning. J Neurosci 26:6314-6317. CrossRef Medline

Fino E, Deniau J-M, Venance L (2008) Cell-specific spike-timingdependent plasticity in GABAergic and cholinergic interneurons in corticostriatal rat brain slices. J Physiol 586:265-282. Medline

Fino E, Paille V, Deniau J-M, Venance L (2009) Asymmetric spike-timing dependent plasticity of striatal nitric oxide-synthase interneurons. Neuroscience 160:744-754. CrossRef Medline

Graybiel AM (1998) The basal ganglia and chunking of action repertoires. Neurobiol Learn Mem 70:119-136. CrossRef Medline

Graybiel AM (2005) The basal ganglia: learning new tricks and loving it. Curr Opin Neurobiol 15:638-644. CrossRef Medline

Groenewegen HJ, Berendse HW (1994) The specificity of the "nonspecific" midline and intralaminar thalamic nuclei. Trends Neurosci 17:52-57. CrossRef Medline

Haber SN (2011) Neuroanatomy of reward: a view from the ventral striatum. In: Neurobiology of sensation and reward (Gottfried JA, ed), Chap 11. Boca Raton, FL: CRC

Haber SN, Calzavara R (2009) The cortico-basal ganglia integrative network: the role of the thalamus. Brain Res Bull 78:69-74. CrossRef Medline

Haber SN, Fudge JL, McFarland NR (2000) Striatonigrostriatal pathways in primates form an ascending spiral from the shell to the dorsolateral striatum. J Neurosci 20:2369-2382. Medline

Haber SN, Kim K-S, Mailly P, Calzavara R (2006) Reward-related cortical inputs define a large striatal region in primates that interface with associative cortical connections, providing a substrate for incentive-based learning. J Neurosci 26:8368-8376. CrossRef Medline

Heidbreder CA, Groenewegen HJ (2003) The medial prefrontal cortex in the rat: evidence for a dorso-ventral distinction based upon functional and anatomical characteristics. Neurosci Biobehav Rev 27:555-579. CrossRef Medline

Ilinsky IA, Jouandet ML, Goldman-Rakic PS (1985) Organization of the nigrothalamocortical system in the rhesus monkey. J Comp Neurol 236: 315-330. CrossRef Medline

Joel D, Weiner I (1994) The organization of the basal ganglia- 
thalamocortical circuits: open interconnected rather than closed segregated. Neuroscience 63:363-379. CrossRef Medline

Kasanetz F, Riquelme LA, Della-Maggiore V, O’Donnell P, Murer MG (2008) Functional integration across a gradient of corticostriatal channels controls UP state transitions in the dorsal striatum. Proc Natl Acad Sci U S A 105:8124-8129. CrossRef Medline

Kincaid AE, Wilson CJ (1996) Corticostriatal innervation of the patch and matrix in the rat neostriatum. J Comp Neurol 374:578-592. CrossRef Medline

Kolb B (1984) Functions of the frontal cortex of the rat: a comparative review. Brain Res 320:65-98. Medline

Koós T, Tepper JM (1999) Inhibitory control of neostriatal projection neurons by GABAergic interneurons. Nat Neurosci 2:467-472. CrossRef Medline

Kremer JR, Mastronarde DN, McIntosh JR (1996) Computer visualization of three-dimensional image data using IMOD. J Struct Biol 116:71-76. CrossRef Medline

Mailly P, Haber SN, Groenewegen HJ, Deniau J-M (2010) A 3D multimodal and multi-dimensional digital brain model as a framework for data sharing. J Neurosci Methods 194:56-63. CrossRef Medline

Mallet N, Le Moine C, Charpier S, Gonon F (2005) Feedforward inhibition of projection neurons by fast-spiking GABA interneurons in the rat striatum in vivo. J Neurosci 25:3857-3869. CrossRef Medline

McGeorge AJ, Faull RL (1989) The organization of the projection from the cerebral cortex to the striatum in the rat. Neuroscience 29:503-537. CrossRef Medline

Middleton FA, Strick PL (1994) Anatomical evidence for cerebellar and basal ganglia involvement in higher cognitive function. Science 266:458461. CrossRef Medline

Nisenbaum ES, Wilson CJ (1995) Potassium currents responsible for inward and outward rectification in rat neostriatal spiny projection neurons. J Neurosci 15:4449-4463. Medline

Parent A (1990) Extrinsic connections of the basal ganglia. Trends Neurosci 13:254-258. CrossRef Medline

Paxinos G, Watson C (1986) The rat brain in stereotaxic coordinates. San Diego, CA: Academic.

Planert H, Szydlowski SN, Hjorth JJ, Grillner S, Silberberg G (2010) Dy- namics of synaptic transmission between fast-spiking interneurons and striatal projection neurons of the direct and indirect pathways. J Neurosci 30:3499-3507. CrossRef Medline

Reep RL, Cheatwood JL, Corwin JV (2003) The associative striatum: organization of cortical projections to the dorsocentral striatum in rats. J Comp Neurol 467:271-292. CrossRef Medline

Roberts AC, Tomic DL, Parkinson CH, Roeling TA, Cutter DJ, Robbins TW, Everitt BJ (2007) Forebrain connectivity of the prefrontal cortex in the marmoset monkey (Callithrix jacchus): an anterograde and retrograde tract-tracing study. J Comp Neurol 502:86-112. CrossRef Medline

Schilman EA, Uylings HB, Galis-de Graaf Y, Joel D, Groenewegen HJ (2008) The orbital cortex in rats topographically projects to central parts of the caudate-putamen complex. Neurosci Lett 432:40-45. CrossRef Medline

Uylings HB, Groenewegen HJ, Kolb B (2003) Do rats have a prefrontal cortex? Behav Brain Res 146:3-17. CrossRef Medline

Van De Werd HJ, Uylings HB (2008) The rat orbital and agranular insular prefrontal cortical areas: a cytoarchitectonic and chemoarchitectonic study. Brain Struct Funct 212:387-401. CrossRef Medline

Voorn P, Vanderschuren LJ, Groenewegen HJ, Robbins TW, Pennartz CM (2004) Putting a spin on the dorsal-ventral divide of the striatum. Trends Neurosci 27:468-474. CrossRef Medline

Wright AK, Norrie L, Ingham CA, Hutton EA, Arbuthnott GW (1999) Double anterograde tracing of outputs from adjacent "barrel columns" of rat somatosensory cortex. Neostriatal projection patterns and terminal ultrastructure. Neuroscience 88:119-133. CrossRef Medline

Yeterian EH, Van Hoesen GW (1978) Cortico-striate projections in the rhesus monkey: the organization of certain cortico-caudate connections. Brain Res 139:43-63. CrossRef Medline

Yin HH, Knowlton BJ (2006) The role of the basal ganglia in habit formation. Nat Rev Neurosci 7:464-476. CrossRef Medline

Zheng T, Wilson CJ (2002) Corticostriatal combinatorics: the implications of corticostriatal axonal arborizations. J Neurophysiol 87:1007-1017. Medline

Zou KH, Warfield SK, Bharatha A, Tempany CM, Kaus MR, Haker SJ, Wells WM 3rd, Jolesz FA, Kikinis R (2004) Statistical validation of image segmentation quality based on a spatial overlap index. Acad Radiol 11:178189. CrossRef Medline 